realities of a later period as well as the conditions in which the utterances were initially spoken.

2. Scholars continue to compare prophetic texts of one culture with those from other settings. For example, recent publication of Akkadian texts attests the preserving of more than one oracle on a clay tablet. Such practice marks the transition from archival preservation of one oracle to the creation of prophetic literature, i.e., the arranged composition of multiple oracles and sayings. Such compositions are observable in both biblical and extrabiblical texts.

3. Study of the rhetorical and literary features of prophetic texts is currently of great interest, particularly as such study has been informed by new understandings of Hebrew poetry.
See also: Akkadian; Bible.

\section{Bibliography}

Blenkinsopp J (1996). A history of prophecy in Israel. Louisville: Westminster John Knox.

Lewis I (1975). Ecstatic religion: an anthropological study of shamanism and spirit possession. Baltimore: Penguin.

Nissinen M (2000). Prophecy in its ancient Near Eastern context: Mesopotamian, biblical, and Arabian perspectives. Atlanta: Society of Biblical Literature.

Overholt T (1989). Channels of prophecy: the social dynamics of prophetic activity. Minneapolis: Fortress.

Petersen D (2002). The prophetic literature: an introduction. Louisville: Westminster John Knox.

Wilson R (1980). Prophecy and society in ancient Israel. Philadelphia: Fortress.

\title{
Propositional and Predicate Logic: Linguistic Aspects
}

P A M Seuren, Max Planck Institute for

Psycholinguistics, Nijmegen, The Netherlands

(c) 2006 Elsevier Ltd. All rights reserved.

\section{Introduction}

Logic is defined by two key notions, entailment and calculus. More precisely, a logic is a calculus for the derivation of entailments. Entailment is a relation between assertive sentences or, rather, between propositions expressed by assertive sentences. (Henceforth, the term 'sentence' is used for 'assertive sentence.') Entailment for sentences is defined as follows:

A sentence or a set of sentences $P$ entails a sentence $\mathrm{Q}(\mathrm{P} \models \mathrm{Q})$ just in case whenever $\mathrm{P}$ is true, $\mathrm{Q}$ must of necessity also be true, on account of the meanings of $\mathrm{P}$ and $\mathrm{Q}-$ that is, for analytical reasons.

There is an immediate complication, however. Strictly speaking, it is not sentences that are true or false but, rather, their utterance tokens and their underlying propositions. It is impossible to say whether the English sentence Jack arrived late is true or false as long as the person called Jack and the time referred to are not specified. The vast majority of natural language sentences are occasion sentences in the sense that they contain a number of parameters that must be given specific values in the world spoken about before there can be talk of truth or falsity. The values for such parameters in one sentence together we call the 'key' of the sentence in question. Only a properly keyed sentence can function as an utterance token with a truth value. It is true that there are sentences, called 'eternal sentences,' that can do without a specific key, such as all humans are mortal. These are to be considered marginal cases whose key is the universe as a whole. Starting with Aristotle, the logical tradition has preferred to avoid the complications caused by the keying condition and has, in principle, developed logics only for eternal sentences. Only recently has there been a greater awareness that such a limitation is not fruitful.

Since the vast majority of sentences are occasion sentences, the entailment relation, when defined for sentences, must be defined modulo key - that is, with reference values kept constant. Under this condition it can be said that Jack has been killed entails Jack is dead because it is in the meaning of the predicate be killed that whoever has been killed is of necessity dead. Propositions, by contrast, if taken to be token occurrences of mental acts in which a property is assigned to one or more objects, are keyed by definition and thus do not need the modulo key condition. The analyticity condition remains unchallenged: When a proposition $\mathrm{P}$ entails a proposition $\mathrm{Q}$, it does so in virtue of the cognitively defined meanings of the predicates in any linguistic expressions of $\mathrm{P}$ and $\mathrm{Q}$.

The analyticity condition must be clearly distinguished from other conditions that justify the drawing of a conclusion. For example, Jack was shot in the heart does not entail Jack died because, even though Jack's death is a direct consequence of his being shot in the heart, this is not a semantic but a physical 
consequence, brought about not by the semantic description of the predicates in question but by physical causation. To be able to draw the corresponding conclusion we need world knowledge, not knowledge of the language.

Most entailments are intuitive in that they are based on speakers' knowledge of their language. Some entailments, however, can be made formal in that they can be computed with the help of a calculus. When that is the case, we have a logic. Every logical system distinguishes between its logical constants and its variables. In propositional calculus, for example, the logical constants are the words and, or, if, and not and the variables range over linguistically expressed propositions. In standard first-order predicate calculus, the logical constants are those of propositional calculus plus the words all and some, and the variables range over predicates and individuals. The logical constants define the calculus.

The notion of logic was discovered in Plato's Academy, mainly by Aristotle (384-322 B.C.E.), who began as a student and later became a prominent member of the Academy. He discovered that the negation word not functions differently according to whether the subject term is or is not quantified. The sentence Plato does not live in Athens is true whenever Plato lives in Athens is false, and vice versa, the former sentence being equivalent to 'it is not true that Plato lived in Athens.' Here, the negation is full sentential, or external, negation. But in, for example, some Greeks do not live in Athens, the negation cannot be external because both this sentence and some Greeks live in Athens can be true at the same time. The difference is that in the latter pair of sentences the subject term is quantified, whereas in the former pair it is a referring expression. Apparently, in sentences with a referring expression as subject term it makes no truth-conditional difference whether the negation is external or internal, but in sentences with a quantified subject term it does. This discovery intrigued Aristotle and some of his fellow students. It eventually resulted in what is now known as AristotelianBoethian Predicate Calculus (ABPC), a form of predicate logic due in the first place to Aristotle but remodeled (and to some extent spoiled) by later commentators, especially the Roman-Christian philosopher Boethius (ca. 475-524 C.E.).

\section{Predicate Calculus}

ABPC, like standard modern logic, is restricted by a few overarching principles. First there is the Principle of Contradiction, which says that no sentence can be true and false at the same time. Then comes the Principle of Bivalence, which says that every sentence
Table 1 Boethius's sentence types

\begin{tabular}{ll}
\hline A: All $F$ is $G$ & E: All $F$ is not- $G \equiv$ No $F$ is $G$ \\
I: Some $F$ is $G$ & O: Some $F$ is not- $G \equiv$ Not all $F$ is $G$ \\
\hline
\end{tabular}

is either true or false, with nothing in between and no further truth values. A further restriction, applying throughout, is the Principle of Extensionality, which restricts the applicability of logic to really existing, actual objects, eliminating all virtual objects (see Virtual Objects), such as Sherlock Holmes or the Golden Mountain, which have been thought up by creative minds.

The Principle of Contradiction seems firmly rooted in cognition and language, but the other two principles, although universally accepted nowadays, are open to doubt. Presuppositional phenomena, in particular, strongly suggest that the Bivalence Principle does not fit the facts of language (see Presupposition). And the Principle of Extensionality is clearly inadmissible in any logico-semantic analysis of human language, which quantifies with equal ease over actual and virtual objects (see Virtual Objects). This last complication is allowed to rest in the present context.

Under the principles given, the (external) negation $n o t$, or $\neg$, is a toggle between truth values: if $\mathrm{P}$ is true, $\neg \mathrm{P}$ is false, and if $\neg \mathrm{P}$ is true, $\mathrm{P}$ is false; $\neg \neg \mathrm{P}$ is equivalent to $\mathrm{P}(\neg \neg \mathrm{P} \equiv \mathrm{P})$. $\mathrm{P}$ and $\neg \mathrm{P}$ are called Contradictories. Given the principles mentioned and the definition of not $(\neg)$ just given, it follows that when $\mathrm{P} \models \mathrm{Q}$, then $\neg \mathrm{Q} \models \neg \mathrm{P}-$ an entailment schema known as Contraposition.

As regards ABPC specifically, Boethius distinguished four sentence types, the types A and I (from Latin affirmo 'I affirm') and $\mathrm{E}$ and $\mathrm{O}$ (from Latin nego, 'I deny'), defined as in Table 1 (the variables $F$ and $G$ range over predicates). ABPC is then defined by the following entailment schemata. Sentences of type $\mathbf{A}$ entail sentences of type $\mathbf{I}$, and those of type $\mathbf{E}$ entail those of type $\mathrm{O}$. Formally: $\mathbf{A} \models \mathbf{I}$ and $\mathbf{E} \models \mathrm{O}$. These are known as the subaltern entailments. Moreover, both $\mathbf{I}$ and $\mathbf{O}$ entail 'there is at least one $F-$ an entailment known as existential import. Given the entailment $\mathbf{A} \models \mathbf{I}$, it follows that $\mathbf{A}$ and $\neg \mathbf{I}$ cannot be true simultaneously, although they may be false together: they are contraries. Aristotle himself added the entailments from some $F$ is $G$ to not all $F$ is not$G$ and from all $F$ is not- $G$ to no $F$ is $G$ (Kneale and Kneale, 1962: 57). However, his later commentators, especially Boethius, went further and added the converses of these two entailment schemata: from not all $F$ is not- $G$ to some $F$ is $G$ and from no $F$ is $G$ to all $F$ is not-G, thereby creating the now standard conversions that allow conversion of all into some, and vice 


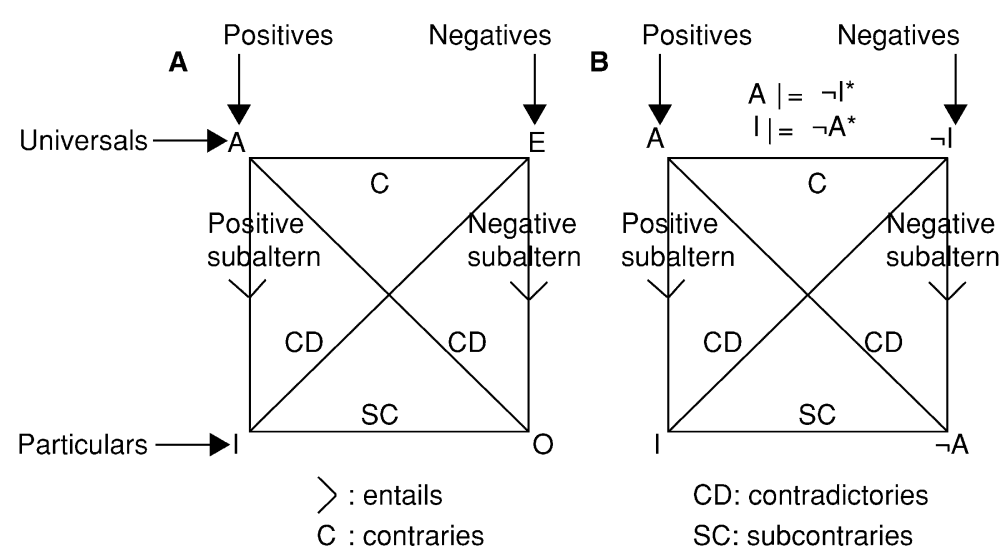

Figure 1 (A) ABPC and (B) AAPC represented as Squares of Oppositions.

versa, provided both an external and an internal negation are added (or removed). Now all $F$ is $G$ and no $F$ is not-G, and some $F$ is $G$ and not all $F$ is not- $G$ are equivalent. With the conversions added, it follows that $\mathbf{I}$ and $\mathbf{O}$ cannot be false together, although they can be simultaneously true; they are subcontraries.

ABPC is known in the traditional form (due to Boethius) of the Square of Oppositions, shown in Figure 1A. Aristotle's original system, called Aristotelian-Abelardian predicate calculus ( AAPC), is shown in Figure 1B (the asterisk stands for internal negation: $\mathrm{A}^{*}$ is all $F$ is not-G; $\mathrm{I}^{*}$ is some $F$ is not-G).

It is important to insist on the difference between ABPC and AAPC because ABPC contains a logical fault that is absent in AAPC. This fault becomes visible when we consider a world (such as ours) without any mermaids. In such a world, the sentence all mermaids swim must be either true or false, owing to the Bivalence Principle. If it is true, some mermaids swim must also be true (subaltern), from which it follows, contrary to fact, that there are mermaids (existential import). But if it is false, then not all mermaids swim is true and entails (by conversion) some mermaids do not swim, which likewise entails (by existential import) that there are mermaids, again contrary to fact. Therefore, all mermaids swim can be neither true nor false when there are no mermaids, which violates the Bivalence Principle. The problem with ABPC is, thus, that it cannot deal with situations in which the F-class is null: only when the F-class is nonnull are the subaltern entailments applicable. This goes against the very notion of logic because entailments are valid not in virtue of world contingencies but in virtue of meanings. This logical fault is called undue existential import (UEI).

To repair this fault, standard modern predicate calculus (SMPC) was developed by mathematical logicians such as Boole, Frege, Peano, and Russell during the late 19 th and early 20 th centuries. SMPC is indeed free from this, or any other, logical fault and is now universally accepted as the one and only sound predicate calculus, which must be embodied in human language if that can lay claim to logical soundness. This deeply ingrained belief, however, is unwarranted.

To see what is at issue it is useful to go back into history. That ABPC is logically faulty was not entirely unknown to medieval philosophers. Peter Abelard (1079-1142), in particular, had seen the fault and restored Aristotle's predicate logic to its original form (hence the name AAPC; see Seuren, in preparation). Yet his (and Aristotle's) way of avoiding UEI was never taken up by the logical tradition, which stuck to the faulty Boethian system until the advent of modern logic. Had the tradition been more attentive, it would have seen not only that the AristotelianAbelardian system is logically sound but also that it has much greater logical power than SMPC. This question is worth unraveling.

Despite its long tradition, the Square of Oppositions is not a very good way of representing ABPC because it expresses neither the conversions nor the role of internal negation. A better representation is shown in Figure 2A, where the symbols $\mathrm{E}$ and $\mathrm{O}$ have been eliminated in favor of external $(\neg)$ and internal (*) negation. The analogous representation of AAPC is given in Figure 2B. Figures $2 \mathrm{~A}$ and $2 \mathrm{~B}$ show two logically isomorphic triangles, each with the vertices A, I, and $\neg \mathbf{I}$, one with and one without the internal negation. In each triangle, the three logical relations are entailment, contradiction, and contrariety. The figures differ in that Figure 2A has the conversions, whereas Figure 2B has the one-way entailments of AAPC, and AAPC lacks the relation of subcontrariety.

A valuation space representation (Van Fraassen, 1971) shows why logical relations hold. The valuation space (VS) /P/ of a sentence $\mathrm{P}$ is the set of situations, in the universe of situations $\mathrm{U}$, in which $\mathrm{P}$ is 
A

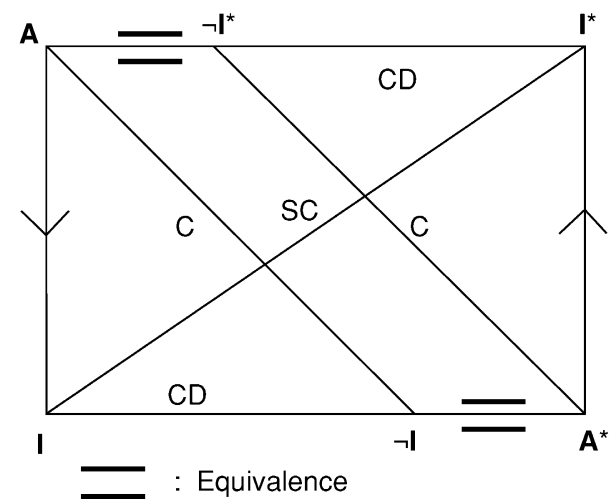

B

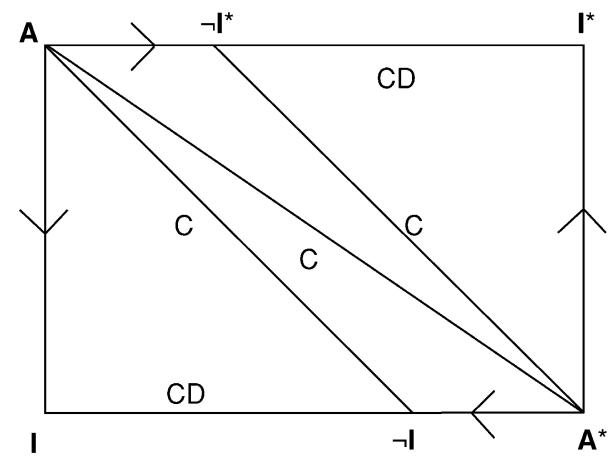

Figure 2 Improved representations of (A) ABPC and (B) AAPC.
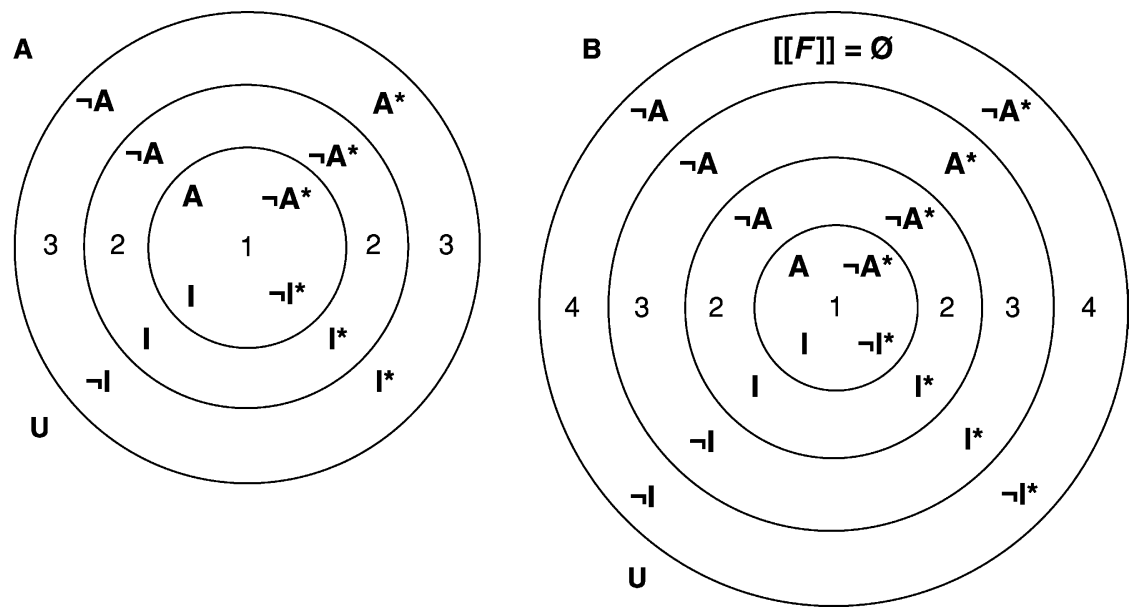

Figure 3 The VS models for (A) ABPC and (B) AAPC

true (modulo key). Logical relations can be read from VS models:

When $/ \mathrm{P} / \subseteq / \mathrm{Q} /, \mathrm{P} \models \mathrm{Q}$.

When $/ \mathrm{P} /=/ \mathrm{Q} /, \mathrm{P} \equiv \mathrm{Q}$.

When $/ \mathrm{P} / \cap / \mathrm{Q} /=\varnothing, \mathrm{P}$ and $\mathrm{Q}$ are contraries.

When $/ \mathrm{P} / \cup / \mathrm{Q} /=\mathrm{U}, \mathrm{P}$ and $\mathrm{Q}$ are subcontraries.

When $/ \mathrm{P} / \cap / \mathrm{Q} /=\varnothing$ and $/ \mathrm{P} / \cup / \mathrm{Q} /=\mathrm{U}, \mathrm{P}$ and $\mathrm{Q}$ are contradictories.

$\mathrm{ABPC}$ is represented in Figure $3 \mathrm{~A}$ and AAPC in Figure 3B.

ABPC lacks a space for cases in which the extension of the $F$-predicate is null $([[F]]=\varnothing)$, which makes its applicability dependent on the contingent condition that $[[F]] \neq \varnothing$, but AAPC has space 4 for the situations in which $[[F]]=\varnothing$. In these situations, the four sentence types, $\mathbf{A}, \mathbf{I}, \mathbf{A}^{*}$, and $\mathbf{I}^{*}$ are all deemed false.

The VSs of the eight sentence types are read from Figure 3 as shown in Table 2 . In both systems, the subalterns hold from $A$ to I because $\{1\} \subseteq\{1,2\}$, and from $A^{*}$ to $I^{*}$ (or, for AAPC, $\neg \mathbf{A}$ ) because $\{3\} \subseteq\{2,3\}$ (or $\{2,3,4\}$ ). But AAPC lacks the subaltern from $\neg \mathbf{I}$ to I* because $\{3,4\} \nsubseteq\{2,3\}$. The conversions do not hold in AAPC because $\neg \mathbf{I}^{*} \not \neq \mathbf{A}$, as $\{1,4\} \nsubseteq\{1\}$ and $\neg \mathbf{I} \not \forall \mathbf{A}^{*}$, as $\{3,4\} \nsubseteq\{3\}$. The contraries have remained intact in AAPC, except for the pair $\neg \mathbf{I}$ and $\neg \mathbf{I}^{*}$, because $\{3,4\} \cap\{1,4\} \neq \varnothing$. Subcontrariety between $\mathbf{I}$ and $\mathbf{I}^{*}$ has been lost in AAPC because $\{1,2\} \cup\{2,3\} \neq \mathbf{U}$.

SMPC presents a solution to UEI based on a direct interpretation of all as set-theoretic inclusion and of some as set-theoretic intersection: all $F$ is $G$ is interpreted as $[[F]] \subseteq[[G]]$, and some $F$ is $G$ as $[[F]] \cap$ $[[G]] \neq \varnothing$. When $[[F]]=\varnothing$, all $F$ is $G$ is true because the null set $\varnothing$ is included in any set. $\mathbf{A}$ and $\mathbf{A}^{*}$ sentences are thus taken to be true when $[[F]]=\varnothing$, which allows the conversions to remain intact. This solution, which depends on the interpretation of all as inclusion and of some as intersection, may be convenient for the logic of mathematics but lacks any linguistic necessity. Given Boolean algebra and standard set theory, we are free to interpret all and some in different terms. For example, all may be defined as yielding truth on condition that $[[F]] \subseteq[[G]]$ and that $[[F]] \neq \varnothing$. This, in fact, appears to correspond more closely to the way the meaning of all is defined in natural language than the simple condition that $[[F]]$ 
Table 2 VS Models of eight sentence types

\begin{tabular}{llll}
\hline ABPC & \multicolumn{3}{l}{ AAPC } \\
\hline$/ \mathbf{A} /$ & $=\{1\}$ & $/ \mathbf{A} /$ & $=\{1\}$ \\
$/ \mathbf{l} /$ & $=\{1,2\}$ & $/ \mathbf{l}$ & $=\{1,2\}$ \\
$/ \mathbf{A}^{\star} /$ & $=\{3\}$ & $/ \mathbf{A}^{*} /$ & $=\{3\}$ \\
$/ \mathbf{I}^{*} /$ & $=\{2,3\}$ & $/ \mathbf{I}^{*} /$ & $=\{2,3\}$ \\
$/ \neg \mathbf{A} /$ & $=\{2,3\}$ & $/ \neg \mathbf{A} /$ & $=\{2,3,4\}$ \\
$/ \neg \mathbf{I} /$ & $=\{3\}$ & $/ \neg \mathbf{I} /$ & $=\{3,4\}$ \\
$/ \neg \mathbf{A}^{*} /$ & $=\{1,2\}$ & $/ \neg \mathbf{A}^{*} /$ & $=\{1,2,4\}$ \\
$/ \neg \mathbf{I}^{*} /$ & $=\{1\}$ & $/ \neg \mathbf{I}^{*} /$ & $=\{1,4\}$ \\
\hline & & & \\
\hline
\end{tabular}

$\subseteq[[G]]$, which fits the facts of language badly. The VS model of SMPC is as in Figure 4.

This shows that almost all entailment relations are lost: the subalterns are gone, as are the contraries and subcontraries. All that has remained is the contradictories and the conversion equivalences. This makes SMPC extremely impoverished compared with either ABPC or AAPC.

It also makes SMPC redundant for the use of language because, when $[[F]]=\varnothing$, the truth value of all eight sentence types considered follows automatically from the theorems of standard set theory. This is so because, for any sets $\mathrm{X}$ and $\mathrm{Y}, \mathrm{X} \subseteq \mathrm{Y}$ when $\mathrm{X}=\varnothing$, and $\mathrm{X} \cap \mathrm{Y}=\varnothing$ when $\mathrm{X}$ or $\mathrm{Y}$ or both equal $\varnothing$. When the class of situations where $[[F]]=\varnothing$ is excluded from SMPC, the truth value of the eight sentence types becomes contingent on circumstances, which makes the corresponding sentences informative. Because the logic that results when the situations where $[[F]]=\varnothing$ are eliminated is identical to ABPC, natural language has a functional interest in ABPC. Such an elimination affects only the semantics of all: $\mathbf{I}$ and $\mathbf{I}^{*}$ sentences are unaffected by the condition that $[[F]] \neq$ $\varnothing$. It follows that if ABPC can be upheld without the logic becoming unsound, the information value of expressions containing the quantifier all will be greatly enhanced. The question is whether natural language can manage to maintain ABPC while keeping its logic sound.

In general, if the semantic description of certain words turns out to be such that they allow for a logical calculus, then that calculus is the logic of language. To find out what the logic of language amounts to, we should, therefore, investigate the meanings of possible logical constants and see what calculus results. For predicate calculus, the logic of language is thus defined by the linguistically given lexical meanings of the words all, some, and not.

It seems that language has outsmarted the logicians by making sentences context-sensitive, using the device of presupposition (see Presupposition), which restricts the informative use of sentences to consistent contexts, in which the presuppositions of the

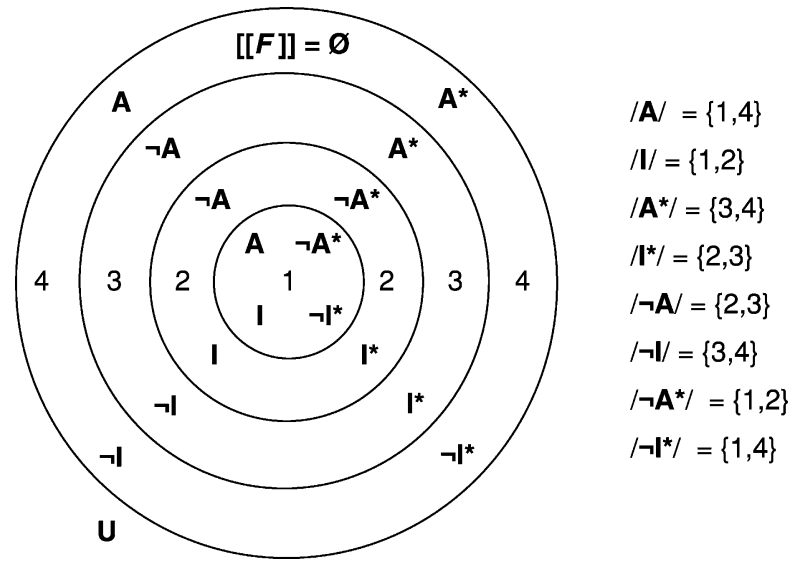

Figure 4 The VS model for SMPC.

sentences in question are stored as preliminary information. The truth of a sentence co-depends on the truth of its presuppositions as stored in the embedding context. If they are not all true, the sentence is false in a special way, called 'radically falsity,' which implies the falsity of at least part of the embedding context and, hence, the futility of the sentence itself in that context. Radical falsity (F2, written as $\simeq$ ) constitutes a third truth value in addition to truth $(\mathrm{T})$ and minimal falsity (F1, written as $\sim$; see Multivalued Logics). Thus, if the lexical meaning of all is made to incorporate the condition that $[[F]] \neq \varnothing$ as a precondition for informative use in a consistent context, ABPC is allowed to apply again in full force, but only within the confines of a situation in which $[[F]] \neq \varnothing$. In situations that do not satisfy that condition, the use of all will result in radical falsity. Although no such provision is required for the lexical meaning description of some, as previously mentioned, $\mathbf{I}$ and $\mathrm{I}^{*}$ sentences are still radically false when $[[F]]=\varnothing$ because the Principle of Extensionality amounts to the presuppositional requirement of actual existence for the reference values of the terms of the $G$-predicate. This presuppositional predicate calculus (PPC) is represented in Figure 5, where the bold circle delineates the area of applicability of traditional bivalent ABPC. Space 4 is useful not only as a logical safety net but also in speech to mark a sentence as being unfit for the current discourse. This is done with the help of the radical negation NOT, emphatically accented, which makes itself useful in language as a metalinguistic discourse corrector, as in all mermaids do NOT swim; there ARE no mermaids!

It thus seems that SMPC should be rejected as a candidate for the status of 'logic of language.' This in itself is a momentous conclusion given the almost universal, yet false, belief that no other consistent predicate logic is possible. There is, however, a redeeming feature. Had it not been for SMPC, the 


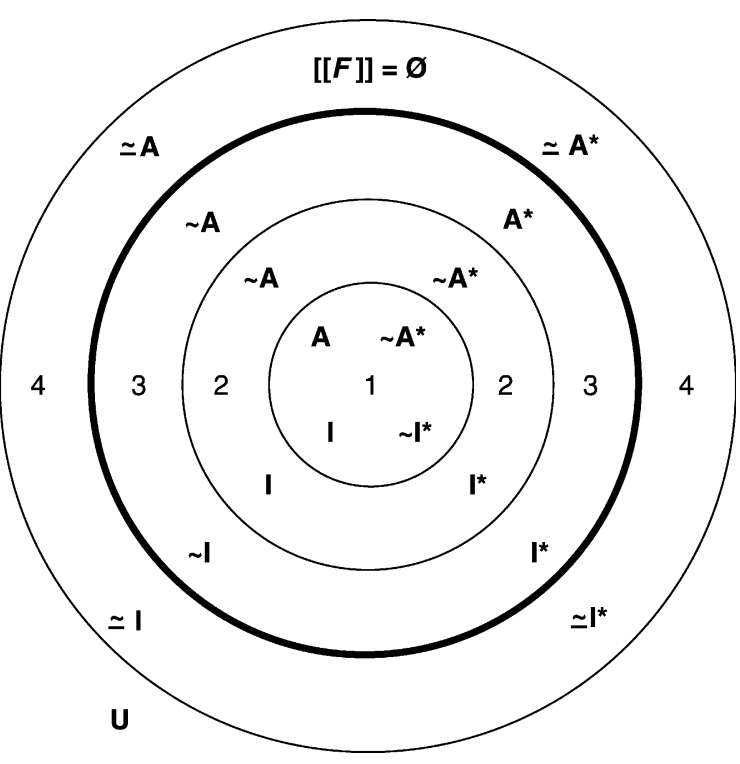

Figure 5 The VS model for PPC.

logical language of predicate calculus ( $\left.\mathrm{L}_{\mathrm{PC}-\mathrm{Peano}}\right)$ in which it is formulated would not have existed. After a first unsuccessful attempt at a logical language in Frege (1878), PL $_{\mathrm{PC}}$ was created by Giuseppe Peano around 1900 and adopted by Alfred Whitehead and Bertrand Russell in their Principia Mathematica of 1910-1913. Since then, L $\mathrm{PC}_{\mathrm{PC} \text {-Peano }}$ has become the stock in trade of all logic.

The main innovation of $\mathrm{L}_{\mathrm{PC}-\mathrm{Peano}}$ consisted in the introduction of separate symbols for all $(\forall)$ and some $(\exists)$ as variable binders in propositional functions. A propositional function is a propositional sentence in $\mathrm{L}_{\mathrm{PC}-\text { Peano }}$ with at least one term missing - that is, filled by a variable. Thus, if Bald(Jack) is a propositional sentence with a truth value, $\operatorname{Bald}(x)$ is a propositional function that will be true or false when a value is filled in for the variable $x$. Quantifiers such as $\forall$ and $\exists$ are a further way of turning a propositional function into a propositional sentence. The universal quantifier $\forall$ turns a propositional function into a sentence with a truth value, in that it requires for truth that any arbitrary value for $x$ (within the defined range of $x$ ) will yield truth. The existential quantifier $\exists$ produces truth when at least one value for $x$ yields truth. A sentence such as All bumans are mortal is rendered in $\mathrm{L}_{\mathrm{PC}-\mathrm{Peano}}$ as (1a), and Some bumans are mortal is rendered as (1b). The symbol $\rightarrow$ stands for the material implication 'if ... then', and $\wedge$ stands for the conjunction 'and'. Both full propositional sentences and propositional functions can be negated and placed under any binary propositional operator.

(1a) $\forall x(\operatorname{Human}(x) \rightarrow \operatorname{Mortal}(x))$ ('for all $x$, if $x$ is human, $x$ is mortal') (1b) $\exists x(\operatorname{Human}(x) \wedge \operatorname{Mortal}(x))$

('there is an $x$ such that $x$ is human and $x$ is mortal')

Example (1a) is true when any arbitrary value for $x$ makes it true. If the value of $x$ is not human but, say, a tree, then the whole implication is automatically true. If for all buman values of $x$ it turns out that they are also mortal, then (1a) is true. Falsity for (1a) can thus be brought about only by one or more values for the variable $x$ that are human but not mortal - so-called counterexamples. For the truth of $(1 \mathrm{~b})$, it is required that there be at least one $x$ such that $x$ is both human and mortal.

$\mathrm{L}_{\mathrm{PC}-\text { Peano }}$ makes it possible to treat sentences with more than one argument term and to quantify each such term. Thus, a sentence such as Every boy gave some present to some girl can be rendered as (2) (disregarding tense):

$$
\begin{aligned}
& \text { (2) } \forall x(\operatorname{Boy}(x) \rightarrow \exists y(\operatorname{Present}(y) \wedge \exists z(\operatorname{Girl}(z) \wedge \operatorname{Give}(x, \\
& y, z)))) \\
& \text { ('for all } x \text {, if } x \text { is a boy then there is a } y \text { such that } y \\
& \text { is a present and such that there is a } z \text { such that } z \\
& \text { is a girl and } x \text { gives } y \text { to } z \text { ') }
\end{aligned}
$$

For the analysis of quantification in natural language sentences, however, this 'translation' method is now widely considered inadequate, mainly because it cannot be used for quantifiers such as most or half. For this reason, Barwise and Cooper (1981) decided to adopt the notion of generalized quantifier, introduced in Mostovski (1957). The actual logic, in the sense of a system of entailment schemata, is not affected by this innovation, which is merely syntactic. In the syntax of this modified logical language $\mathrm{L}_{\mathrm{PC}-M o s t o v s k i}$, a quantifier is a binary higher order predicate - that is, a binary predicate over sets, not over individuals. Instead of writing (1a) and (1b), we now write (3a) and (3b), respectively; (2) is now rendered as $(3 \mathrm{c})$ :

(3a) $\forall x(\operatorname{Human}(x), \operatorname{Mortal}(x))$

('the set of humans is included in the set of mortals')

(3b) $\exists x(\operatorname{Human}(x), \operatorname{Mortal}(x))$ ('there is a nonnull intersection of the set of humans and the set of mortals')

(3c) $\forall x(\operatorname{Boy}(x), \exists y(\operatorname{Present}(y), \exists z(\operatorname{Girl}(z)$, Give $(x, y$, to $z)))$ )

('the set of boys is included in the set of those individuals $a$ such that for each $a$ there is a nonnull intersection of the set of presents and the set of objects $b$ such that there is a nonnull intersection of the set of girls and the set of persons that $a$ gives $b$ to')

$\mathrm{L}_{\mathrm{PC}-M o s t o v s k i}$ fits both the syntactic and the semantic facts of language remarkably well and is now widely 
used in philosophical, semantic, and logical studies of language. It is important to realize that the syntax of a logical language is to a large extent independent of the precise semantics of the quantifiers and the negation: The language may stay the same while the semantic description of the operators involved varies, and vice versa. The main question regarding the logic of language is now no longer the syntax of the logico-semantic 'translations' of sentences because $\mathrm{L}_{\mathrm{PC}-\text { Mostovski }}$ has so far proved adequate. The main problem still outstanding is, rather, the precise semantic description of whatever logical constants are detected, in particular the words not, all, some, and, or, and if. (May (can) and must are likewise plausible candidates for the status of logical constants in a modal logic, and other candidates may yet be discovered.) So far, the syntax of $\mathrm{L}_{\mathrm{PC}-\text { Mostovski }}$ has proved highly successful not only as regards the expression of the logical properties of propositions, but also for the purpose of relating its expressions to surface sentences in natural languages. It has vastly extended the scope of the logical analysis of natural language sentences and is probably the greatest contribution made by modern logic to the logico-semantic analysis of natural language.

\section{Propositional Calculus}

In propositional calculus, the most current logical constants are not, and, or, and if ... then. The
A

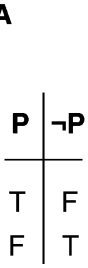

Figure 6
B

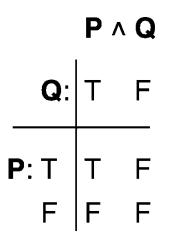

C

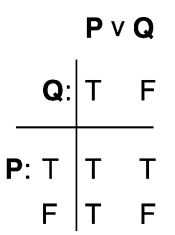

D

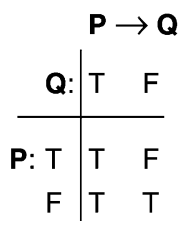

variables, say $P, Q, R, \ldots$, range over propositional sentences. Propositional calculus was developed in the Greek world by Stoic philosophers during the 3rd and 2nd centuries B.C.E. (Kukasiewicz, 1934). Not is standardly symbolized as $\neg$ and is the bivalent truth-value toggle described here. And is standardly written as $\wedge$, or as $\vee$, and if ... then as $\rightarrow$. The semantics of these constants (operators) is usually given in terms of the truth tables shown in Figure 6.

Strictly speaking, the material implication operator $\rightarrow$ is superfluous because $\mathrm{P} \rightarrow \mathrm{Q} \equiv \neg \mathrm{P} \vee \mathrm{Q}$. Yet it is usually specified as a separate operator rendering natural language if ... then. That it does not do so has been known for two millennia, yet it continues to figure in logic textbooks as a translation of if ...then.

Of greater logical importance is the fact that the logic of and and or is isomorphic with the logic of all and some in ABPC. When $\mathrm{P} \wedge \mathrm{Q}$ stands for $\mathrm{A}, \mathrm{P} \vee$ $\mathrm{Q}$ for $\mathrm{I}, \neg \mathrm{P} \vee \neg \mathrm{Q}$ for $\mathrm{A}^{*}$, and $\neg \mathrm{P} \vee \neg \mathrm{Q}$ for $\mathrm{I}^{*}$, there is full isomorphism. The following notation is used:

$$
\begin{array}{ll}
\text { AND: } \mathrm{P} \wedge \mathrm{Q} & \text { OR: } \mathrm{P} \vee \mathrm{Q} \\
\neg \text { AND: } \neg(\mathrm{P} \wedge \mathrm{Q}) & \neg \text { OR: } \neg(\mathrm{P} \vee \mathrm{Q}) \\
\text { AND }^{*} \neg \mathrm{P} \wedge \neg \mathrm{Q} & \text { OR* }: \neg \mathrm{P} \vee \neg \mathrm{Q} \\
\neg \text { AND }^{*}: \neg(\neg \mathrm{P} \wedge \neg \mathrm{Q}) & \neg \mathrm{OR}^{*}: \neg(\neg \mathrm{P} \vee \neg \mathrm{Q})
\end{array}
$$

Propositional calculus can now be represented as the Square of Oppositions of Figure 7A. The VS model for propositional calculus is as in Figure $7 \mathrm{~B}$, which is isomorphic with Figure 3A. The 'conversions' in predicate calculus correspond to the $D e$ Morgan's laws in propositional calculus; they convert $\wedge$ into $\vee$ and vice versa, provided both an external and an internal negation are added (or removed): $\neg(\mathrm{P} \wedge \mathrm{Q}) \equiv \neg \mathrm{P} \vee \neg \mathrm{Q}$, and $\neg(\mathrm{P} \vee \mathrm{Q}) \equiv \neg \mathrm{P} \wedge \neg \mathrm{Q}$.

It has often been observed that the universal quantifier is somehow related to and and the existential quantifier to or. This relation is usually spelled out by saying that all $F$ is $G$ is equivalent to ' $G\left(f_{1}\right) \wedge G\left(f_{2}\right) \wedge$ $G\left(f_{3}\right) \wedge \ldots$, and that some $F$ is $G$ is equivalent with
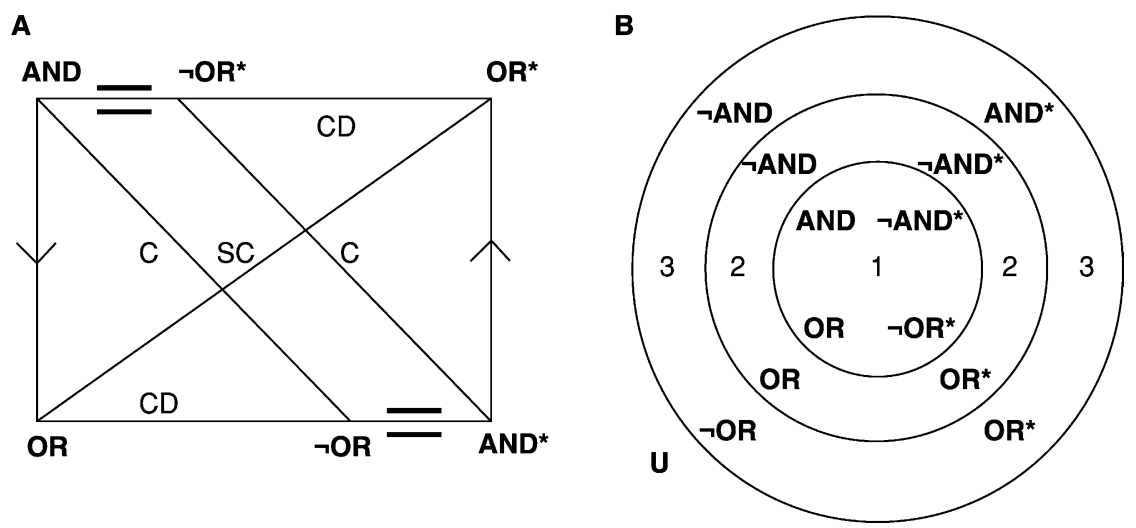

Figure 7 (A) Square and (B) VS model for standard propositional calculus. 
A

\begin{tabular}{l|l|l}
$\mathbf{P}$ & $\sim \mathbf{P}$ & $\approx \mathbf{P}$ \\
\hline $\mathbf{T}$ & $\mathrm{F} 1$ & $\mathrm{~F} 1$ \\
$\mathrm{~F} 1$ & $\mathrm{~T}$ & $\mathrm{~F} 1$ \\
$\mathrm{~F} 2$ & $\mathrm{~F} 2$ & $\mathrm{~T}$
\end{tabular}

B

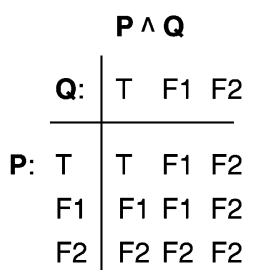

C

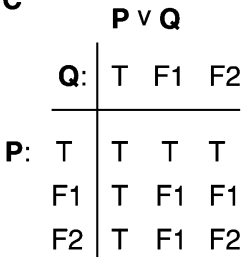

Figure 8 Truth tables for Presuppositional Propositional Calculus (PPC).

' $G\left(f_{1}\right) \vee G\left(f_{2}\right) \vee G\left(f_{3}\right) \vee \ldots$,', until all members $f_{n}$ of $[[F]]$ have been enumerated. Yet this is only so if $[[F]] \neq \varnothing$. If $[[F]]=\varnothing$ there will be neither a conjunction nor a disjunction of its members. In general, since ABPC was rejected in favor of SMPC, which had its logic reduced to the conversions, the obvious parallelism of $\forall$ with $\wedge$ and of $\exists$ with $\vee$ has been more of an embarrassment than of a help to logicians. Yet, if $\mathrm{ABPC}$ can be restored to its traditional position, the isomorphism of ABPC with propositional calculus will be a reason for looking further into logical systems with such properties as ABPC and propositional calculus have in common.

Unlike ABPC, propositional calculus is not logically faulty. It does not need a fourth space because there is no null class to cater for. Yet it can be made trivalent so as to cater for presuppositions (Seuren et al., 2001). The minimal, presupposition-preserving negation $(\sim)$ toggles between T and F1 and yields F2 in cases of presupposition failure. The radical presupposition-canceling negation $(\sim)$ yields T only in cases of presupposition failure and yields F1 otherwise. (The standard negation $\neg$ is equivalent with the disjunction of minimal and radical negation: $\sim \mathrm{P} \vee \simeq \mathrm{P} \equiv \neg \mathrm{P}$.) Conjunction $(\wedge)$ selects $F 2$ over F1 and $T$, and F1 over $\mathrm{T}$; disjunction $(\mathrm{V})$ selects $\mathrm{T}$ over $\mathrm{F} 1$ and $\mathrm{F} 2$, and F1 over F2, as shown in Figure 8.

When the logic of language is seen as being defined by the semantic descriptions of the logical constants involved, an entirely new perspective on logic emerges, one that has so far hardly been explored.

See also: Aristotle and Linguistics; Extensionality and Intensionality; Multivalued Logics; Presupposition; Virtual Objects.

\section{Bibliography}

Barwise J \& Cooper R (1981). 'Generalized quantifiers and natural language.' Linguistics and Philosophy 4(2), 59-219.

Frege G (1878). Begriffsschrift. Eine der arithmetischen nachgebildete Formelsprache des reinen Denkens. Halle: Nebert.

Kneale W \& Kneale M (1962). The development of logic. Oxford: Clarendon Press.

Łukasiewicz J (1934). 'Z historii logiki zdán.' Przegląd Filozoficny 37, 417-437. [German translation: 'Zur Geschichte der Aussagenlogik,' Erkenntnis 5, 111-131; English translation: 'On the history of the logic of propositions.' In McCall (1967: 67-87)].

McCall S (ed.) (1967). Polish logic 1920-1939. Oxford: Oxford University Press.

Mostovski A (1957). 'On a generalization of quantifiers.' Fundamenta Mathematica 44, 12-36.

Seuren P A M (in preparation). Mind, logic and language.

Seuren P A M, Capretta V \& Geuvers H (2001). 'The logic and mathematics of occasion sentences.' Linguistics \& Philosophy 24(5), 531-595.

Van Fraassen B (1971). Formal semantics and logic. New York: Macmillan.

\section{Propositional Attitude Ascription: Philosophical Aspects}

L Clapp, Illinois Wesleyan University, Bloomington, IL, USA

(C) 2006 Elsevier Ltd. All rights reserved.

Paradigmatic propositional attitude ascriptions (in English) are sentences of the form A Vs that S, where $A$ is a singular definite noun phrase referring to a rational agent, $\mathrm{S}$ is a sentence, and $\mathrm{V}$ is a propositional attitude verb. Paradigmatic propositional attitude verbs include 'believe', 'doubt,' 'realize,' 'hope,' 'regret,' etc. Such verbs refer to intentional mental states with representational content, where this content can be evaluated for truth or falsity, and thus can be identified with a proposition. (What are sometimes called verbs of saying, e.g., 'say,' 'deny,' 'announce,' are closely related to, but typically distinguished from, propositional attitude verbs.) Because propositional attitude verbs refer to intentional states with propositional content, it is natural to endorse a relational analysis of propositional attitude ascriptions: an assertion of 'John hopes that Mary won' says that the referent of 'John' bears the mental relation (or attitude in Russell's 1940 terminology) referred to by 'hopes' toward the proposition expressed by the complement clause 'that Mary won.' Similarly, an assertion of 